\title{
On the theory of Weber fractions
}

\author{
KENNETH H. NORWICH \\ University of Toronto, Toronto, Ontario, Canada
}

\begin{abstract}
The Weber fraction is treated as part of an information theoretical view of perception. In this theory of sensory perception, subjective magnitude is related to the information transmissible from stimulus to perceiver. The derived psychophysical law can be approximated as a power or logarithmic law, depending on conditions. The mathematical expression for the Weber fraction is obtained as a derivative of the psychophysical law. One of many interesting relationships derived here is that the product of the Stevens exponent with $\log _{10}$ stimulus range, for many sensory modalities, is equal to the maximum information transmitted per stimulus (in bits, measured in a test of categorical judgments) multiplied by 0.6 . This number is a derived constant and is not measured from the data.
\end{abstract}

If we represent the physical magnitude of a stimulus by $\Phi$ and the change in physical magnitude of the stimulus corresponding to one just noticeable difference $(\mathrm{jnd})^{1}$ by $\Delta \Phi$, then $\Delta \Phi / \Phi$ is often known as a Weber fraction. The subjective magnitude of the stimulus, as determined by the method of magnitude estimation, will be represented by $\Psi$. The Weber fraction has been measured for many different modalities of sensation over a wide range of values of $\Phi$. The relationship between $\Delta \Phi / \Phi$ and $\Phi$ is usually of the form shown in Figure 1: the fraction begins high for small values of $\Phi$, and then falls with increasing $\Phi$ to something approximating a plateau. Occasionally it is found to rise again, above the plateau, at high values of $\Phi$ (not shown in the figure).

In this paper, we shall develop a general theory of the Weber fraction. We shall obtain a mathematical expression for $\Delta \Phi / \Phi$ that accounts for the observed shape of the curve in Figure 1; and we shall be able to predict the parameters of the curve on theoretical grounds. As an unexpected bonus from the theory, we shall be able to derive a general mathematical relationship connecting the Stevens exponent, $n$, with the range of physical magnitudes, $\Phi$, spanned by a given modality. The theoretical function for the Weber fraction that we shall obtain is derived quite simply by differentiating an expression for the "psychophysical law." The theory developed herein will be examined in historical context. The Weber fraction, Fechner's law, Stevens's law, receptor adaptation, and many other psychophysical phenomena, find unity within this information theoretical description of the process of perception.

To follow the derivations in the present work, it is necessary first to gain an appreciation of how the psychophysical law, or the law of sensation, may be interpreted through the medium of information theory. These ideas were developed in a series of papers (Norwich 1977,

This work was supported by a grant from the Natural Sciences and Engineering Research Council of Canada. The author's mailing address is Institute of Biomedical Engineering, University of Toronto, 4 Taddle Creek Road, Toronto, ON M5S 1A4, Canada.
$1978,1981 \mathrm{a}, 1981 \mathrm{~b}, 1982,1983,1984)$. The reader who is familiar with even the first and fourth of these papers may wish to proceed immediately to the section on Derivation of the Weber Fraction from the Psychophysical Law.

\section{The Information Content of a Steady Stimulus From an Experiment on Categorical Judgments}

It was not many years after the appearance of the seminal papers by Shannon (1948) that information theory was applied in the analysis of experiments on perception. Garner and Hake (1951) demonstrated how to calculate $H_{m}$, the maximum information transmissible by a steady stimulus, from the stimulus-response matrix generated when a subject makes categorical judgments of a number of stimuli.

From the known probabilities, $p_{i}$, governing the administration of stimuli in each category, the stimulus information, $H_{s}$, may be calculated from the equation

$$
H_{s}=-\sum_{i} p_{i} \log p_{i}
$$

Since the subject does not perform flawlessly, $H_{s}$ is not equal to the transmitted information, $H_{m}$, but must generally be diminished by the "stimulus equivocation," $H_{e}$. The equivocation may be calculated from a generalization of Equation 1, which allows for the presence of conditional probabilities. Thus we have

$$
H_{m}=H_{s}-H_{e} \text {. }
$$

If $H_{m}$ is measured in bits of information, then $2^{H_{m}}$ gives us a measure of the maximum number of categories that can be identified without error. This value is largely independent of the number of categories (beyond a certain minimum) into which the stimulus continuum was divided. Henceforth, we shall refer to $H_{m}$ as the "information content of the stimulus." $H_{m}$ was found to assume a relatively constant value across a number of sensory modalities. We shall take its mean value to be about 2.5 bits of information per stimulus. 


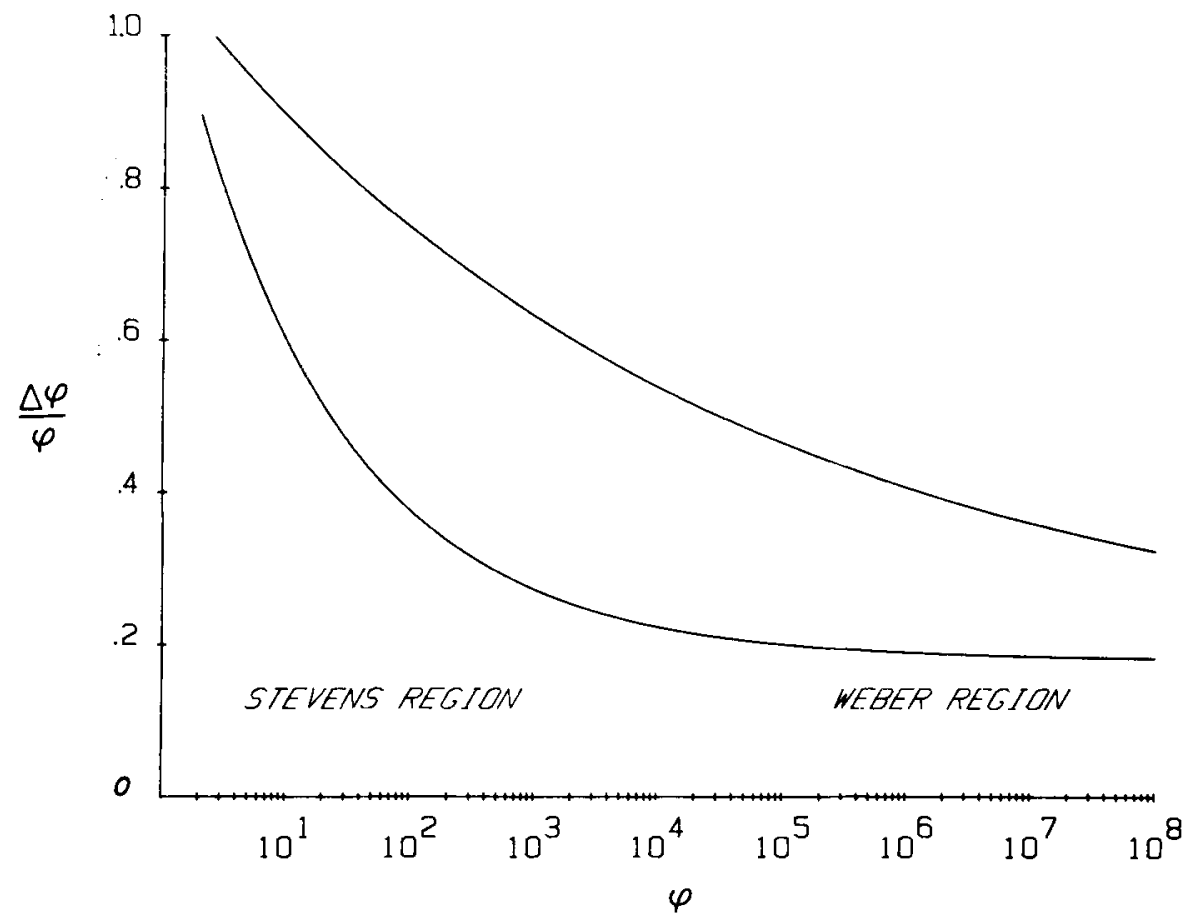

Figure 1. The general appearance of the graph of the Weber fraction, $\Delta \Phi / \Phi$, plotted against stimuIus intensity, $\Phi$. Lower curve reaches a plateau; upper curve does not. For taste of salinity and for brightness, the fraction tends to rise slightly at larger $\Phi$. Weber and Stevens regions are discussed in the text.

A more thorough treatment of many of the above topics is given by Attneave (1959) and Garner (1962).

\section{The Entropic or Informational}

\section{Theory of Perception}

First premise. From information theory we learn that the uncertainty (entropy) that precedes the occurrence of an event is reduced after observation of the outcome of the event. The vanished entropy is captured as information. The fundamental premise of this paper and of each member of the group that preceded it is that perceptual activity involves such a process of entropy reduction and, moreover, that perception can occur only when informational entropy is reduced (Norwich, 1977). However, since entropy encodes the magnitude of uncertainty, we might ask how this uncertainty arises in the process of sensory perception.

One possibility is that it is due to microscopical fluctuations in the density of the stimulus. To gain an appreciation of this, let us consider, by way of illustration, the perception of a taste stimulus (Norwich, 1984).

We shall consider only steady stimuli, by which is meant stimuli that are macroscopically uniform rather than changing with time. So we shall be concerned with the process of tasting a solution (say a dilute solution of glucose) that remains in contact with the tongue. However, it is important to realize that although the macroscopical concentration of the stimulating solution is unchanging (that is, the fractional change per second in the number of solute molecules in each milliliter of solution is vanishingly small), at the microscopical level the concentration of solute is changing substantially on a moment-to-moment basis because of random movements of the solute molecules. This is illustrated schematically in Figure 2. The large boxes contain an ensemble of molecules. The ensemble consists of 15 molecules at both times $t_{1}$ and $t_{2}$, showing that the concentration, or density, of molecules is macroscopically constant. The small boxes represent
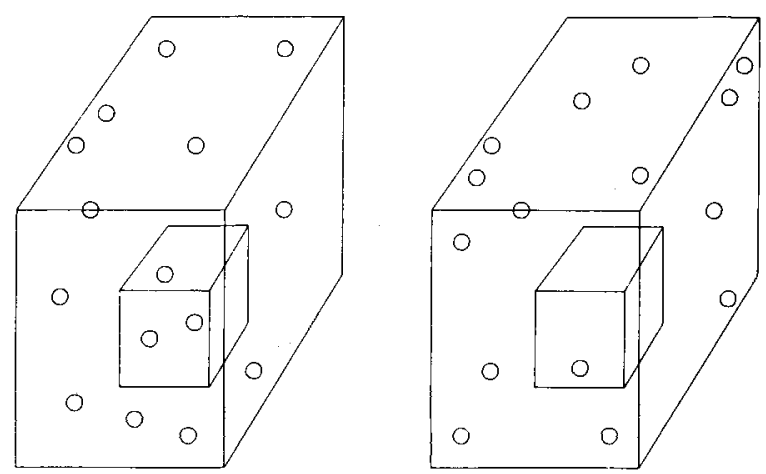

Figure 2. Illustration of the concept that a stimulus may be macroscopically constant (unchanging in time), but microscopically nuctuating. The boxes on the left-hand side represent a dilute solution at time $t_{1}$ and on the right-hand side at time $t_{2}$. The large boxes contain identical numbers of particles (15); the small boxes contain different numbers ( 3 and 1$)$. 
a microscopical taste receptor. The receptor samples 3 molecules at $t_{1}$ but only one molecule at $t_{2}$, due to the random fluctuations in molecular configuration. Since a taste receptor operates at the microscopical level, it will, of necessity, experience a fluctuating concentration, or density, of solute molecules. (Similarly a photoreceptor will experience a fluctuating photon density in a macroscopically constant light stimulus, etc.) Now, the organism subserved by the taste receptor, the human perceiver for example, is concerned not with the moment-by-moment fluctuations in concentration, but with the prevailing, or mean, concentration of the stimulus. That is, by assumption, the microscopically structured receptor is concerned with reporting the mean stimulus intensity as inferred from a sequence of fluctuating microscopical samples. Receptor uncertainty at this most elemental level, then, can be taken to mean uncertainty about the mean intensity of a steady stimulus as inferred from individual samples of the stimulus.

How can we utilize receptor uncertainty to calculate sensory information? We cannot employ Equation 1 directly, because the quantities $p_{i}$ are discrete probabilities and, in the analysis of sensory signals such as concentration, $c$, of solutions we must manage probability densities such as $p(c)$, where the quantity $p(c) d c$ plays the role of a probability. It is tempting, by analogy to Equation 1, to represent $H$, the sensory information, by the integral,

$$
H=-\int p(c) \log p(c) d c .
$$

However, this is not correct. This expression is known as differential entropy and is not adequate, in itself, to express information. One can show, for example, that varying the units in which concentration, $c$, is measured will change the value of $H$. To overcome this problem, when using probability densities as opposed to discrete probabilities, we must calculate information from the difference between two differential entropies:

$$
H=-\int p_{s r} \log p_{s r} d c+\int p_{r} \log p_{r} d c .
$$

The probability density $p_{r}$ is often referred to as noise; it is a signal that originates within the receptor and, by combining with the true external stimulus signal, limits the resolution of the receptor, and hence its capacity to relay information. This "noise," however, seems necessary if we are to quantify the amount of information transmitted, so the subscript $r$ has been adopted to designate a reference input. The probability density $p_{s r}$ is that of the pure external signal combined (convoluted) with the reference or noise signal, and represents the total input. When the stimulus input (pure external signal) is sufficiently weak so that $p_{s r} \rightarrow p_{r}$, it is seen, from Equation 4 , that the information transmitted is equal to zero.

Let us now proceed to evaluate $H$ in Equation 4. By a straightforward process of integration, it can be shown that if the probability density is Gaussian or normal with variance $\sigma^{2}$, the value of the differential entropy defined by Equation 3 is $\left(1 / 2 \ln \left(2 \pi e \sigma^{2}\right)\right.$. We shall assume that the reference input is governed by the normal distribution with variance $\sigma_{r}^{2}$, and hence that its differential entropy equals $(1 / 2) \ln \left(2 \pi e \sigma_{r}^{2}\right)$. That is,

$$
-\int p_{r} \log p_{r} d c=(1 / 2) \ln \left(2 \pi e \sigma_{r}^{2}\right) .
$$

This is the value of the second integral in Equation 4. What can we now say about the first integral in Equation 4?

We may not, in general, know the nature of the probability density governing the stimulus input (pure external signal) but, by conjecture, it is not information about this "raw" fluctuating signal that is required, but, rather, information about the mean of a number of samplings made by the receptor of its stimulus signal. By the central limit theorem of statistics, the means of samples of size $m$ from a population with arbitrary distribution and variance $\sigma^{2}$ will tend to lie on a normal distribution with variance $\sigma^{2} / m$. Let the variance of the stimulus input be $\sigma_{s}^{2}$. When the receptor has made $m$ samplings from its stimulus signal, it may estimate a single mean of sample size $m$, which will tend to form part of a normal distribution with variance $\sigma_{s}^{2} / m$. This distribution must be combined or convoluted with the reference distribution whose variance is $\sigma_{r}^{2}$. By a straightforward integration process, we find that the combined distribution, $p_{s r}$, for the signal mean (with variance $\sigma_{r}^{2} / m$ ) with the reference (with variance $\sigma_{r}^{2}$ ) is again the normal distribution with variance $\sigma_{r}^{2} / m+\sigma_{r}^{2}$. The differential entropy is now found to be

$$
-\int p_{s r} \log p_{s r} d c=(1 / 2) \ln \left[2 \pi e\left(\sigma_{s}^{2} / m+\sigma_{r}^{2}\right)\right] \text {. }
$$

Introducing Equations 5 and 6 into Equation 4, we can finally evaluate the entropy $H$ :

$$
H=(1 / 2) \ln \left(1+\sigma_{s}^{2} / m \sigma_{r}^{2}\right), \quad m \geq 1 .
$$

It is important to appreciate, qualitatively, the significance of the $H$ function given by Equation 7 . We deal with a macroscopically steady stimulus. $\sigma_{s}^{2}$ is the variance of densities in the stimulus population (a measure of molecular fluctuation, in the case of the sense of taste). $m$ is the number of samplings made by the receptor of its stimulus at any time during the process of perception, and therefore, $m$ is also the size of one sample of the mean. $\sigma_{r}^{2}$ is a constant property of the receptor. $H$ then gives the "uncertainty" of the receptor about the mean of the population of densities (mean concentration when dealing with taste) as inferred from one sample of size $m$. $H$, expressed by Equation 7 in natural units, is an entropy, a residual uncertainty, or the amount of information which, in principle, could yet be obtained by the receptor after it has made $m$ samplings.

Second premise. The second premise can be stated as follows: The "perceptual variable" is proportional to the receptor entropy. In earlier papers (Norwich 1977, 1978, 1981a), the perceptual variable was taken to be frequency of impulses, $F$, in the primary afferent neuron in a dissected preparation of receptor plus neuron. That is,

$$
F=k^{\prime} H \text {. }
$$


In later work and in the present paper, the perceptual variable is taken as the subjective magnitude of a stimulus. That is,

$$
\Psi=k H,
$$

where $k$ is a constant which is greater than zero.

Equation 9 is not a trivial extension of Equation 8. Some of the justifications for making the extension have been discussed by Norwich (1984). There is some evidence that, for the sense of taste, the subjective magnitude is directly proportional to impulse frequency in afferent neurons mediating taste; that is, $\Psi \propto F$, which will give Equation 9 from Equation 8. This is the sort of result one would expect if sensory receptors were behaving "in parallel," with no collateral influence on each other. Then the neural report of each receptor to higher cerebral centers would be identical, and one might expect that the magnitude of sensation would parallel the impulse frequency in each contributing neuron. The above circumstance is, however, very idealistic. Perhaps it is best to take refuge in the purely pragmatic approach: Regard Equation 9 as a premise that stands in place of Equation 8 . If the equations resulting from its adoption are valid, this may justify the premise.

\section{The Psychophysical Law as an Equation of Entropy}

Let us now convert Equation 7 into more familiar forms. It is know from statistical physics that the mean value of the density (concentration) of a population of particles, $\Phi$, is often (but not invariably) related to the variance of the population, $\sigma^{2}$, by a relation of the form

$$
\sigma^{2}=\lambda \Phi^{n},
$$

where $n$ is a constant, characteristic of the species of particle.

Equation 10 is an expression of the fact that measurements of larger quantities are generally associated with greater errors or fluctuations than measurements of smaller quantities. If a receptor samples its stimulus to gain knowledge of the constitutent molecular density, it will encounter a fluctuation in its density measurements that is proportional to the square root of the mean molecular density. Hence, variance varies as mean density to the first power. For photons, the issue is, in principle, a little more complex. Einstein's equation gives the variance for photon density as a quadratic function of the mean. However, the Wien approximation is used in vision research (Barlow, 1964), in which, again, variance varies as the mean to the first power.

The exponent $n$ in Equation 10 relates $\Phi$ and $\sigma^{2}$ in a physical stimulus. However, the same value of $n$ will not necessarily relate $\Phi$ in the stimulus, where it is measured by the experimenter, with $\sigma^{2}$ at the sensory receptor. For example, displacements of the basilar membrane in the cochlea are not related even in a linear manner to sound pressure levels at the eardrum (Rhode, 1971). Thus, the value of $n$ "seen" or appreciated by the hair cell will differ from that obtained from the density of the air. Undoubtedly, fluctuations from other sources will also reach the hair cells.

We can return now to Equation 7 armed with the approximate relation

$$
\sigma_{s}^{2}=\lambda \Phi^{n},
$$

where the value of $n$ will usually not be known to us a priori. For a constant value of $m$, we can combine Equations 7,9 , and 11 to obtain

$$
\Psi=(1 / 2) k \ln \left(1+\beta \Phi^{n}\right),
$$

where $\lambda / m \sigma_{r}^{2}$ has been condensed into a single constant, $\beta$. Equation 12 then gives $\Psi$, the subjective magnitude of a stimulus, as a function of $\Phi$, the (mean) magnitude of the physical stimulus.

This equation states that the subjective magnitude of a stimulus is proportional to the amount of "uncertainty" experienced by the perceiver about the mean of its stimulus. It is a measure of the perceiver's potential for receiving information. It tells us how much information can still be received, or stated another way, it is the maximum information still transmissible to the perceiver. $\Psi$ increases with the physical magnitude, $\Phi$, because population variance, $\sigma_{s}^{2}$ (measuring fluctuation), increases with $\Phi$ (Equation 11).

When $\beta \Phi^{n}$ is much larger than unity, Equation 12 may be approximated by a simpler and more familiar form:

$$
\begin{aligned}
& \Psi=(1 / 2) k \ln \left(\beta \Phi^{n}\right) \\
& \Psi=(1 / 2) k \ln \beta+(1 / 2) k n \ln \Phi
\end{aligned}
$$

or simply

$$
\Psi=A_{1} \log \Phi+A_{2} ; \quad A_{1}, A_{2} \text { constants. }
$$

We recognize Equation 13 as Fechner's law, and we understand now that it is expected to be valid only in the region where the quantity $\beta \Phi^{n}$ is large.

When $\beta \Phi^{n}$ is smaller than unity, $\ln \left(1+\beta \Phi^{n}\right)$ in Equation 12 can be approximated by means of a Taylor expansion. From fundamental calculus,

$$
\ln (1+z)=z-z^{2} / 2+z^{3 / 3}-\cdots
$$

For sufficiently small values of $z$, we can write

$$
\ln (1+z)=z
$$

to a good approximation. Introducing this approximation into Equation 12, we obtain another familiar form,

$$
\Psi=(1 / 2) k \beta \Phi^{n},
$$

or simply

$$
\Psi=\alpha \Phi^{n} ; \quad \alpha \text { constant, }
$$

which we recognize as a form of Stevens's law. Again, the value of $\beta \Phi^{n}$ determines the range of valid approximation.

There is good evidence in the recent literature to support the concept that Fechner's and Stevens's laws are, 
in effect, approximations of the more general form of the psychophysical law given by Equation 12 . For example, Atkinson (1982) showed examples of the deviation of experimental data from the simple power law for larger values of $\Phi$. It can easily be seen that these deviations (data fall below the expected straight line) are approximately taken into account by using the first two terms in the Taylor series (Equation 14) rather than just the first. From McBride (1983a, see especially Figure 4), it can also be seen that experimental data may deviate from Fechner's law for smaller values of $\Phi$ (data fall above the expected straight line). This will occur because the approximation given by Equation 13 weakens for smaller $\Phi$.

In summary of the issue of power versus logarithmic laws: a small value of $\beta \Phi^{n}$ favors a power law, and a large value favors a logarithmic law. Since $\beta \Phi^{n}=\sigma_{s}^{2} / m \sigma_{r}^{2}$, and $\sigma_{r}^{2}$ is regarded as constant, the value of $\beta \Phi^{n}$ is governed by the ratio of pure signal variance, $\sigma_{s}^{2}$, to the number of samplings, $m$, made by the receptor of its signal population. Therefore, both a signal of large amplitude (intense stimulus) and fewer samplings (brief stimulus duration) will favor a logarithmic law, whereas both a signal of smaller amplitude (less intense stimulus) and a greater number of samplings (more prolonged stimulus and hence partially adapted state) will favor a power law.

We can recognize, now, that Fechner's and Stevens's laws are each approximations to a more general form of the psychophysical law, which is, in reality, the law governing the transmission of sensory information (Equation 12).

\section{Information Content of a Steady Stimulus From Adaptation Data}

We recall that $m$ was the number of samplings made by a receptor of its stimulus population. Let us take $m$, to a first approximation, as proportional to the time, $t$, during which the stimulus has been applied. If we introduce this feature into Equation 7, and combine the new Equation 7 with Equations 9 and 11 as before, we obtain, as a more readily applicable version of Equation 12,

$$
\begin{gathered}
\Psi=(1 / 2) k \ln \left(1+\beta^{\prime} \Phi^{n} / t\right), \\
t \geq t_{0}=\text { time of first sampling. }
\end{gathered}
$$

Let us consider now an experiment to test the change in subjective magnitude, $\Psi$, with time, $t$ (adaptation). Since the stimulus magnitude, $\Phi$, is kept constant, Equation 16 can be written more simply in the form

$$
\Psi=(1 / 2) k \ln (1+B / t), \quad B \text { constant } \quad t \geq t_{0}
$$

Since $\Psi=k H$ (from Equation 9), $\Psi$, therefore, describes the progressive decrease in entropy or uncertainty associated with continual sampling of the stimulus. In other words, the familiar phenomenon of adaptation is interpreted by the entropy theory of perception not as fatiguing of the receptor but as the decrease in receptor uncertainty (gain in certainty) that attends protracted exposure to a stimulus. The longer you observe something, the more sure you are about what you are observing-to a saturable limit.

If we curve-fit Equation 17 to experimental data, we can evaluate the parameters $k$ and $B$. Since $\Psi=k H$, we can divide the experimental values for subjective magnitude, $\Psi$, by the value obtained for $k$, to give the values for entropy, $H$ (Norwich, 1981a).

Now the excursion of $H$ during an experiment on adaptation, that is, $H_{\max }-H_{\min }$, gives the total amount of information transmitted to the receptor during the prolonged period of stimulation. (We recall that reduction of entropy, $H$, is recaptured as information.) But this value must be equal to or greater than the corresponding value for $H_{m}$ determined in an experiment on categorical judgments (Equation 2), since the judgments are limited by the maximum sensory information received. That is,

$$
H_{\max }-H_{\min } \geq H_{m},
$$

where $H_{\min }$ is often quite close to zero. Thus we see that curve-fitting the entropy function to adaptation data permits us to make a new, totally independent measurement of the information content of the stimulus. The two methods (category test and adaptation curve) have always been found to agree closely (Norwich, 1981a, 1984).

The information content of a stimulus will, therefore, be taken to mean the maximum information transmissible per stimulus, measured either by the method of categories or by analysis of adaptation data, since both methods give, effectively, the same number.

Finally, it is only necessary to observe that in any psychophysical experiment, for the largest value of stimulus intensity, $\Phi_{m}$, the corresponding value of $H$ given by

$$
H_{m}=\Psi_{m} / k
$$

will approach the value of $H_{\max }$, the maximum entropy for that sensory modality. Therefore, in the work on Weber fractions that follows, we shall take the value of $H$ corresponding to $\Phi$ values far to the right on the plateau in Figure 1 as approaching $H_{\max }$, and, therefore as an estimate of the maximum information transmissible by that sensory modality.

It should be emphasized that we are dealing here with an approximation: $H \cong H_{\max }$. It is recognized that very many factors affect the measurement of $H_{m}$ and $H_{\max }$ in the laboratory. When we come to test the predictions of the entropy theory in Table 1, the nature and extent of the above approximations must be borne in mind.

\section{Derivation of the Weber Fraction \\ From the Psychophysical Law}

For the remainder of this paper we shall require the law of sensation (psychophysical law) only in its timeindependent form, as given in Equation 12:

$$
\Psi=(1 / 2) k \ln \left(1+\beta \Phi^{n}\right) .
$$

As promised, the mathematical form of Weber's fraction 
follows immediately by differentiating Equation 12 with respect to $\Phi$ :

$$
\frac{d \Psi}{d \Phi}=\frac{(1 / 2) k n \beta \Phi^{n-1}}{1+\beta \Phi^{n}} .
$$

Replacing $d \Psi$ and $d \Phi$ by $\Delta \Psi$ and $\Delta \Phi$, respectively, and rearranging the equation algebraically gives us the Weber fraction, $\Delta \Phi / \Phi$, as a function of stimulus magnitude, $\Phi$ :

$$
\frac{\Delta \Phi}{\Phi}=\frac{2 \Delta \Psi}{n \beta k}\left(\beta+\Phi^{-n}\right) .
$$

The Weber fraction may also be written in the fuller form (fractional change in stimulus magnitude per subjective jnd):

$$
\frac{\Delta \Phi}{\Phi} / \Delta \Psi=\frac{2}{n \beta k}\left(\beta+\Phi^{-n}\right)
$$

Equations 21 and 22, then, give the expression for the Weber fraction that emerges from the informational theory of perception. The curve described by this equation, particularly if $\Delta \Psi$ is taken to be constant, is the one that is classically observed in experiments on difference limens (Figure 1): $\Delta \Phi / \Phi$ is high for low values of intensity, $\Phi$, and tends to fall to a plateau for higher values of $\Phi$ (that is, $\Phi^{-n} \rightarrow 0$ ). We must observe, though, that there is nothing, in theory, that impels $\Phi^{-n}$ to become vanishingly small in the physiological range of $\Phi$. Therefore, in principle, certain modalities of sensation can be expected to show progressively decreasing Weber fractions throughout the entire physiological range of stimulus intensities. The history of Equation 21 and other theoretical approaches to the Weber fraction will be discussed below.

It has been emphasized that the Weber fraction is not constant throughout the full range of stimulus intensities. The plateau region of the curve in Figure 1, if it exists, will be referred to as the "Weber-Fechner region," since in this region we have, from Equation 21 together with Fechner's assumption of constant $\Delta \Psi$,

$$
\frac{\Delta \Phi}{\Phi}=\frac{2}{n k} \Delta \Psi=\mathrm{a} \text { constant, }
$$

an equation which, when integrated, gives the WeberFechner logarithmic law of perception. In the left-hand portion of the curve in Figure 1, $\Delta \Phi / \Phi$ falls sharply with increasing $\Phi$ due to the term $\Phi^{-n}$. Since $n$ is the Stevens exponent, this part of the curve might be called the "Stevens region."

\section{Total Just Noticeable Differences Beneath the Plateau of Weber's Fraction}

As shown by Riesz (1928), the total number of jnds contained within a stimulus of magnitude $\Phi^{\prime}$ is given by the simple expression

$$
\text { Total jnds }=\int_{\Phi_{0}}^{\Phi^{\prime}} \frac{d \Phi}{\Delta \Phi}
$$

where $\Phi_{0}$ is the threshold intensity. The reasoning behind this equation is not difficult to see: $d \Phi / \Delta \Phi$ gives the num- ber of jnds that "fit into" $d \Phi$, a small element of $\Phi$. If we sum the contributions from all elements of $\Phi$ from threshold upward, we obtain the total jnds. Since $d(\ln \Phi) / d \Phi=1 / \Phi$, therefore $d \ln \Phi=d \Phi / \Phi$, or $d \Phi=$ $\Phi d \ln \Phi$. Hence, we can write Equation 24 in the equivalent form

$$
\text { Total jnds }=\int_{\Phi_{0}}^{\Phi^{\prime}} \frac{d \ln \Phi}{\Delta \Phi / \Phi} .
$$

Equation 25 will be found to be very useful, since it relates total jnds directly to the Weber fraction, $\Delta \Phi / \Phi$. Since $\Delta \Phi / \Phi$ is constant beneath the plateau, if we consider only the total number of jnds beneath the plateau, say between the lower bound of the plateau, $\Phi_{p}$, and some maximum, $\Phi_{m}$, we may take $\Delta \Phi / \Phi$ outside the integral sign in Equation 25. That is,

$$
\text { Total jnds beneath plateau }=\left(1 / \frac{\Delta \Phi}{\Phi}\right) \int_{\Phi_{p}}^{\Phi_{m}} d \ln \Phi,
$$

or

$$
\text { Total jnds beneath plateau }=\left(1 / \frac{\Delta \Phi}{\Phi}\right) \ln \left(\Phi_{m} / \Phi_{p}\right) .
$$

Since $\ln x=\ln 10 \cdot \log _{10} x$, Equation 26 may be written

Total jnds beneath plateau

$$
=\frac{\ln 10 \cdot\left(\log _{10} \text { stimulus range }\right)}{\text { Weber constant }}
$$

where " $\log _{10}$ stimulus range" means $\log _{10} \Phi_{m}-\log _{10} \Phi_{p}$, and "Weber constant" means the value of the Weber fraction under the plateau. This is an equation of great generality relating three important psychophysical variables: total jnds beneath the plateau, stimulus range, and the Weber constant.

\section{$\Delta \Psi$, The Subjective Magnitude of One JND}

Stevens (1936) and Miller (1947) attempted to show that $\Delta \Phi$, the subjective magnitude of the jnd, was not constant throughout the range of auditory perception as Fechner had originally conjectured. They reached this conclusion by comparing, as $\Phi$ increased, the growth of total jnds with the growth in subjective magnitude. For audition, $\Delta \Phi$ is smaller for small $\Phi$ than for large $\Phi$. However, McBride (1983b) has recently questioned the variability of $\Delta \Psi$, and suggested that Fechner's original hypothesis of constant $\Delta \Psi$ may, indeed, be correct.

We shall now derive three equations, each relating the maximum information transmissible per stimulus to the Stevens exponent, using the mathematical properties of the Weber fraction. For purposes of these derivations, we shall approximate $\Delta \Psi$ as constant (Fechner's conjecture). It might be expected that this approximation will hold well in the Weber region of the Weber fraction, but will progressively weaken as we move backward into the Stevens region. Below, we shall test the extent of the validity of each of the three equations. 
Relationship Between Information Per Stimulus and the Stevens Exponent

Using the plateau in the Weber region. From Equation 23, we have in the Weber region of Figure 1,

$$
\frac{\Delta \Phi}{\Phi}=\frac{2 \Delta \Psi}{n k}=\text { Weber's constant, }
$$

and from Equation 9

$$
k=\Psi / H .
$$

Hence,

$$
\frac{\Delta \Phi}{\Phi}=\frac{2 H}{n(\Psi / \Delta \Psi)} .
$$

But, since $\Delta \Psi$, the subjective magnitude of one jnd, is constant in the Weber region by assumption, we may interpret $\Psi / \Delta \Psi$ as approximately equal to the total number of jnds making up the subjective magnitude, $\Psi^{2}$ We recall, however, from the final paragraph of the section on information from adaptation data (page 290) that the value of $H$ corresponding to a $\Phi$ value on the extreme right-hand side of the plateau of Weber's fraction will approach $H_{\max }$ and, therefore, the information content of the stimulus (information per stimulus). Hence, by rearranging Equation 28 and converting from natural $\log$ units to bits,

Information per stimulus (bits) $=\frac{1}{2 \ln 2}$

- (Stevens exponent) (Weber constant) (total jnds).

This equation, derived by Norwich (1984) for the sense of taste, is now seen to represent any sensory modality for which Equations 9 and 12 are valid, subject to the additional constraint that $\Delta \Psi$ is taken as constant.

The hyperbola of $\mathbf{R}$. Teghtsoonian. If, for the total number of jnds beneath the plateau, we substitute in Equation 29 the expression given in Equation 27, we obtain the approximate relationship,

Information per stimulus (bits)

$$
=\frac{\ln 10}{2 \ln 2} \text { (Stevens exponent) } \cdot \log _{10} \text { (stimulus range), }
$$

where we recall (see Equation 27) $\log _{10}$ (stimulus range beneath plateau) $=\log _{10} \Phi_{m}-\log _{10} \Phi_{p}=\log _{10} \Phi_{m} / \Phi_{p}$. In fact, the information per stimulus does not vary greatly and, evaluated in experiments on categorical judgments, has a mean value of about 2.5 bits. If we substitute this mean value for the information into Equation 30, we obtain the approximate relationship involving the remaining two variables:

(Stevens exponent)( $\log _{10}$ stimulus range)

$$
=\frac{2.5 \times 2 \ln 2}{\ln 10}=1.51 \text {. }
$$

The constant $2 \ln 2 / \ln 10=2 \log _{10} 2=0.6$ is an invariant governing all sensory modalities, and does not arise from the data.

Teghtsoonian (1971), using data from experiments by Stevens and his colleagues that had been assembled by Poulton (1967), demonstrated the validity of Equation 31 . He found, by curve-fitting, that the exponent and range were related as a rectangular hyperbola,

(Stevens exponent) $\left(\log _{10}\right.$ stimulus range $)=1.53$.

The theoretical value for the constant, 1.51 , is quite close to the measured value of 1.53. Teghtsoonian's graph is reproduced here in Figure 3. I believe that this is the first time the curve has been accounted for on theoretical grounds, and a fortiori, the theory used was not designated specifically for that purpose. The same system of equations that gave rise to Equation 31 has accounted for many other psychophysical phenomena, in the manner discussed in the first six sections.

It should be noted here that the validity of Equations 9 and 12 from which Equation 31 was derived was demonstrated theoretically only for stimuli of the "intensity type" (Norwich, 1977, 1978, 1981a, 1981b, 1982, 1983, 1984). However, Equation 31 has been shown by

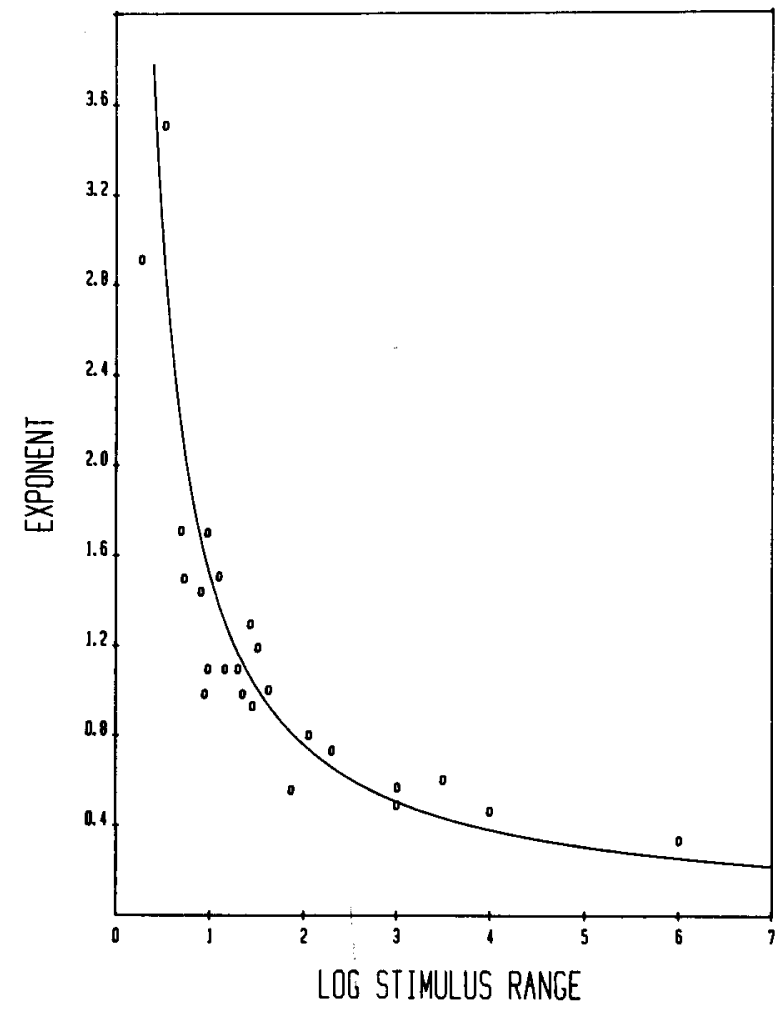

Figure 3. The exponent of the power function is plotted against $\log _{10}$ stimulus range for many sensory modalities. The curve is of the theoretically predicted function (exponent)( $\log _{10}$ stimulus range) $=2.5 \times 2 \times \ln 2 / \ln 10=1.5$. (From "On the Exponents in Stevens' Law and the Constant in Ekman's Law" by R. Teghtsoonian, 1971, Psychological Review, 78, p. 73. Copyright 1971 by American Psychological Association. Reprinted by permission.) 
Teghtsoonian to be valid not only for stimuli of the intensity type, but also for other types of stimuli, such as saturation of yellow or magnitude estimation of fingerspan. Do Equations 9 and 12 hold for other modalities as well?

It must also be conceded, in appraising the validity of Equation 31 that there is a degree of arbitrariness involved in estimating the mean value of the information per stimulus. It depends on which modalities are included in the average. The value of 2.5 bits can be argued to be a little high.

It should also be observed that the Teghtsoonian hyperbola follows directly from Equation 12 without passing through the intermediary of Weber's fraction if it is accepted that over rather a large range of $\Phi$ values the psychophysical law is often described adequately by a logarithmic function as well as by a power function. The adequacy of both functions has been illustrated, for example, by Stevens (1970) for the visual data of Hartline and Graham, and recently by McBride (1983a), who showed that taste data conformed well to a Weber semilog plot (where others have used full-log plots). Equation 30 (the hyperbola) follows directly from Equations 9 and 12 by taking the logarithmic approximation

$$
H=(1 / 2) n \ln \Phi+(1 / 2) \ln \beta \text { (cf. Equation 13). }
$$

Taking $\Phi_{m}$ and $\Phi_{p}$ to be the upper and lower bounds to the Weber region, respectively,

$$
\begin{aligned}
H_{m} & =(1 / 2) n \ln \Phi_{m}+(1 / 2) \ln \beta \\
H_{p} & =(1 / 2) n \ln \Phi_{p}+(1 / 2) \ln \beta .
\end{aligned}
$$

Subtracting one equation from the other,

$$
\begin{aligned}
H=H_{m}-H_{p} & =(1 / 2) n\left(\ln \Phi_{m}-\ln \Phi_{p}\right) \quad \text { (natural units) } \\
& =\frac{n}{2 \ln 2}\left(\ln \Phi_{m}-\ln \Phi_{p}\right) \quad \text { (bits) } \\
& =\frac{\ln 10}{2 \ln 2} n\left(\log _{10} \Phi_{m}-\log _{10} \Phi_{p}\right) \quad \text { (bits). }
\end{aligned}
$$

That is,

Information per stimulus (bits)

$$
=\frac{\ln 10}{2 \ln 2} \text { (Stevens exponent) } \cdot \log _{10} \text { (stimulus range) }
$$

which is Equation 30 and hence Equation 31.

Using the extremes of the Weber fraction. We can avoid the approximation engendered by using only jnds beneath the plateau by using the values of the Weber fraction at the extremes (highest and lowest values) of the stimulus intensity. As derived in the Appendix,

Information per stimulus (bits)

$$
\begin{aligned}
= & \frac{\ln 10}{2 \ln 2} \text { (Stevens exponent) } \log _{10} \text { (stimulus range) } \\
& -\frac{1}{2 \ln 2} \ln \left(\frac{S_{0}}{S_{\infty}}\right),
\end{aligned}
$$

where $S_{0}$ is the value of the Weber fraction, $\Delta \Phi / \Phi$, just above threshold, $S_{\infty}$ is its value for maximum stimulus intensity, and "stimulus range" now refers to the total stimulus range, not just the range beneath the plateau.

Ostensibly this equation is superior to Equations 29, 30 , and 31 , since it utilizes total stimulus range rather than the range spanned by an ill-defined plateau. However, Equation 32 does require our knowing the value of $S_{0}$ accurately, and this may not be possible in practice. Moreover, when $S_{0}$ is large, the approximation of $\mathrm{d} \Phi$ by $\Delta \Phi$ as made in Equation 21 may not be valid.

In the following section, we shall associate the value of information per stimulus calculated from the entropy equation with the corresponding quantity measured in an experiment on categorical judgments. In doing this, one must always bear in mind the constraints discussed in the final paragraph of the section on adaptation data: Information per stimulus in Equations 29 through 32 is an upper bound to the corresponding quantity measured in an experiment on categorical judgments.

\section{Numerical Evaluation of the Three Equations Relating Information Per Stimulus With the Stevens Exponent}

The accuracy of the hyperbolic relationship given by Equation 31 has already been documented by Teghtsoonian (1971) (Figure 3). We shall now provide a preliminary test of the validity of Equations 29, 30, and 32 for each of the senses of taste, vision, and hearing, within the limitations imposed by published data. Preliminary evaluation of Equation 29 for taste has been made by Norwich (1984). The calculations are summarized in Table 1.

In order to evaluate these equations for a number of sensory modalities, it was necessary to delve far back into the literature. One must find sensory systems for which there are careful measurements of all four variables-the exponent of the power function, the Weber fraction in its entirety (which provides the stimulus range), and the maximum information transmitted per stimulus. The only sense for which these criteria were met is taste. Although intensity of visual stimulus and intensity of auditory stimulus are included in Table 1, some of the values entered are approximations. One should recall that the quantity of information transmitted per stimulus, as determined from categorical judgments, $H_{m}$, is roughly the same for all modalities, that is, $2^{H_{m}} \cong$ Miller's "magical number $7 \pm 2$." Thus we could, probably with no real loss in resolution, set $H_{m}$ equal to $\log _{2} 7$ or $\log _{2} 6$ and make all comparisons with this value.

For an evaluation of visual intensity, difference limen data measured by König and Brodhun in the 19th century, as reported by Hecht (1934), were used. The Stevens exponent for brightness is usually reported to be about .33 (Mansfield, 1973). No data on categorical judgments of luminance are available, so the estimate $\log _{2} 6=2.6$ bits of information is used.

For audition of pure tones, a wealth of data was provided by Knudsen (1923) and Riesz (1928). Riesz's results were 
Table 1

Evaluation of the Theoretical Predictions for the Senses of Taste, Vision, and Hearing

\begin{tabular}{lccc}
\hline & Taste (Saline) & Visual Intensity & $\begin{array}{c}\text { Auditory } \\
\text { Intensity }\end{array}$ \\
\cline { 2 - 4 } & (A) EXPERIMENTAL DATA & .33 & $.33^{*}$ \\
Stevens exponent & 1. & 1000. & $184 . \dagger$ \\
Total jnds & 9.7 & 8. & $9 .-10$. \\
$\log _{10}$ (total stimulus range) & 1.9 & 4. & 5. \\
$\log _{10}$ (range below plateau) & 1. & .68 & 2.0 \\
Weber fraction, $S_{0}$ & .90 & .01 & .065 \\
Weber fraction, $S_{\infty}$ & .28 & & \\
Information per stimulus, $H_{m}$, & & 2.6 & 2.51 \\
measured in experiment on & 1.70 & König \& Brodhun & Riesz (1928) \\
categorical judgments & Holway \& & Garner (1953) \\
Sources of above data & Hurvich (1937) & $\begin{array}{l}\text { (pecht, 1934) } \\
\text { (estimated) }\end{array}$ &
\end{tabular}

(B) THEORETICAL PREDICTIONS

$\begin{aligned} & \text { Information per stimulus } \\ & \text { calculated from Equation } 29\end{aligned}$
$\begin{aligned} & \text { Information per stimulus } \\ & \text { calculated from Equation } 30\end{aligned}$
$\begin{aligned} & \text { Information per stimulus } \\ & \text { calculated from Equation } 32\end{aligned}$

presented in exactly the form required by Equation 21 so that they were easily employed. However, Riesz measured the jnd using a method involving beats, rather than the more common method described by Coren, Porac, and Ward (1984). ${ }^{1}$ This may introduce an aberration in our results. Moreover, the parameters of the Weber fraction are a function of the frequency of the tone, which poses some problems. Garner (1953) placed an upper limit of 2.51 bits per stimulus on the information transmitted by the intensity of a pure tone, but did not specify its frequency. I compared the 2.51 bits with difference limen data appropriate for a $512-\mathrm{Hz}$ tone. The number of jnds is a function of the frequency of the tone, as shown in Figure 7 of Riesz's paper. Moreover, Garner's experiment was conducted within the range of $15-110 \mathrm{~dB}$, so that the total number of jnds between these limits was used in Equation 29.

For the sense of taste, the data seem to be reliable and compatible. The mean value of the Stevens exponent for sodium chloride is quite close to 1.0 (Meiselman, Bose, \& Nykvist, 1972; Norwich, 1984). Information transmitted per stimulus was measured by Beebe-Center, Rogers, \& O'Connell (1955). The Weber fraction was measured by Holway and Hurvich (1937). These authors presented their data in both tabular and graphical form, which made them very easy to use.

For vision, the theoretically predicted value from Equation 32 was lower than the "global" value $\log _{2} 6$ bits. The mean deviation of theoretical from measured value of $H_{m}$ was $24 \%$. The predicted and measured values for the sense of taste agree reasonably well except, again, for the value from Equation 32. The mean deviation of theoretical from measured $H_{m}$ was $16 \%$. Predicted and measured values of $H_{m}$ for auditory intensity show reasonable agreement. Mean deviation was $10 \%$.

It should be observed that the use of Equation 30 involved an estimate of the breadth of the plateau of the Weber fraction. Since the plateau is seldom perfectly flat, there is an arbitrary element involved in estimating its breadth. For example, in the lower curve of Figure 1, the range extends from $\Phi=10^{8}$ back to $10^{4}$ or $10^{3}$. Admittedly, this introduces uncertainty of $20 \%-25 \%$ into $\log _{10}$ (range). No such arbitrary element enters into the use of Equations 29 and 32.

In assessing the accuracy of the theoretical predictions in Table 1, the extent of the approximations used must be borne in mind: the association of $H_{\max }$ with $H_{m}$ (Equation 18), the constraint holding $\Delta \Psi$ corresponding to one jnd constant, the approximation of $\Delta \Phi$ by $d \Phi$, the estimation of the width of the plateau and the value of $S_{0}$ from Weber fraction data, inconsistencies in the design of experiments whose results were compounded, and the introduction of data measured with the technology of a century ago. Nonetheless, the general agreement between theoretical prediction of the value of information transmitted and that measured in the laboratory is quite apparent. This general agreement is obtained despite variation by a fac- 
tor of $10^{2}$ in the total number of jnds, and a factor of $10^{8}$ in the range of stimuli. The table is meant to illustrate a trend rather than to give specific numerical results.

Table 1 may be improved as better data become available, or it may be modified to meet the needs of the user. In amending the entries to Table $1 \mathrm{~A}$, one must bear in mind that the data within a column are interdependent. For example, if one replaces the exponent .33 for sound intensity with the exponent .66 for sound pressure, corresponding changes must be made in the stimulus range and the Weber fractions, each of which are halved. Similarly, the Weber fraction curve from which data are drawn for evaluating $S_{0}, S_{\infty}$ must be the same as that used for calculating the total jnds using Equation 24 or Equation 25 (because, for example, if $S_{\infty}$ is found to be half its listed value, total jnds will be found to be about double). The various entries in each column of Table 1A "conspire" or trade off to produce near-constant values for the logarithm of the "magical number" that are entered in Table 1B.

\section{On the Various Theories of the Weber Fraction}

Although Weber originally suggested that the jnd function was of the form $\Delta \Phi / \Phi=$ a constant, it was recognized even from early experiments that this function was not adequate because of the rapid fall in the fraction for low $\Phi$ values. Apparently, Fechner proposed a modification of Weber's function of the form (Luce \& Galanter, 1963)

$$
\frac{\Delta \Phi}{\Phi}=K_{1}+K_{2} / \Phi
$$

We can recognize this as identical in structure to the informational Weber fraction (Equation 21), but with the value of $n$ equal to 1 . In order to describe empirically the process of visual discrimination, Nutting (1907) is reported to have generalized Equation 33 to the form

$$
\frac{\Delta \Phi}{\Phi}=K_{1}+K_{2} / \Phi^{n},
$$

which is identical to the informational form. It was utilized in this form empirically by Knudsen (1923) and Riesz (1928) to describe the differential sensitivity of the ear for pure tones.

It is, incidentally, most instructive to compare Riesz's empirical equation for the Weber fraction (using his symbolism),

$$
\Delta E / E=S_{\infty}+\left(S_{0}-S_{\infty}\right)\left(E_{0} / E\right)^{r},
$$

directly with the theoretical expression, Equation 21 ( $E$ corresponds to $\Phi$ ). Riesz's exponent, $r$, corresponds to the Stevens exponent, $n$. Hence, Riesz's graph of the variation of $r$ with frequency of tone gives the variation of the Stevens exponent with frequency. It can be seen to vary from about .28 to .47 for sound intensity. (See also Appendix.)
Miller (1947) used the restricted form of the Weber fraction (Equation 33), empirically, in his study on sensitivity to auditory noise.

However, possibly the first attempt to derive the form of the jnd function from more elementary equations was made by Ekman (1959), whose paper is the real predecessor of the current work. Ekman began with the power law of sensation in the form

$$
\Psi=c(\Phi+a)^{n} .
$$

He then introduced a restriction, $\Delta \Psi / \Psi=\mathrm{a}$ constant, and by differentiating and rearranging, emerged with the restricted form of the jnd function Equation 33. Deriving the form of the Weber fraction in this way is quite different from citing it empirically. One can see quite clearly why, and under what restrictions, the jnd function emerges from a power law of sensation.

Green and Luce (1974) approached the theory of the Weber fraction for audition from a statistical model of the distribution of interarrival times (IAT) between successive auditory neural pulses. They approximated the IAT distribution by the exponential distribution, and then set the parameter of this distribution equal to a power function of intensity, as suggested to them by reaction time data. Proceeding in this fashion, they succeeded in deriving an expression for the Weber fraction. Parameters were evaluated using Riesz's (1928) data. Their model is highly complex but may be the first that proceeds from a neurophysiological base.

Falmagne's (1974) approach to the Weber fraction for audition is subtle. Defining the discrimination index, $M\left(\Phi_{x}, \Phi_{y}\right)$, as the probability that a subject will judge $\Phi_{x}$ to be louder than $\Phi_{y}$, Falmagne then assumed

$$
M\left(\Phi_{x}, \Phi_{y}\right) \leq M\left(\Phi_{x}^{\prime}, \Phi_{y}^{\prime}\right) \text { iff } \frac{\Phi_{x}^{n}+\varkappa}{\Phi_{y}^{n}+\varkappa} \leq \frac{\Phi_{x}^{\prime n}+\varkappa}{\Phi_{y}^{\prime n}+\varkappa} .
$$

He then proceeded, without further assumption, to derive mathematically an expression for the Weber fraction. However, before evaluating this expression using experimental data, he constrained the discrimination index:

$$
M\left(\Phi_{x}, \Phi_{y}\right)=\text { a constant. }
$$

He then completed his derivation of the Weber fraction in the form

$$
\Delta \Phi / \Phi=\left(B+A / \Phi^{n}\right)^{1 / n}-1,
$$

which conformed reasonably well to experimental data.

A strength of Falmagne's work is its mathematical elegance and the fewness of its assumptions. A shortcoming is that for the data of Knudsen (1923) the fitted value of the exponent $n$ is about .6 rather than the required .3 .

The entropic or informational form of the jnd function (Equation 21) can be compared and contrasted with all of the above. The informational function arises, as did Ekman's, from the psychophysical law of sensation. It is, therefore, more general than those derived specifically for 
one modality. It does not issue from a neurophysiological base, as does the model of Green and Luce. It is a theoretical equation, like Ekman's, but emerging now from the very fundamental principle that the information received by a perceiver cannot exceed that transmitted from the stimulus. It is not an empirical equation like that of Fechner, Knudsen, and others. There is one strong constraint applied $(\Delta \Psi=$ a constant for the jnd), similar in severity to those applied by Ekman and Falmagne. However, this constraint may be relaxed in future refinements of the informational equation (see next section). The strength of the informational jnd equation is that it forms part of a network of equations that describe many psychophysical effects. Moreover, the values of constants predicted by the informational equation have generally been confirmed experimentally (e.g., the constant in Teghtsoonian's hyperbolic function, and the values of Riesz's $r$ parameter).

\section{Conclusions and Projections}

Fundamentally, this paper has dealt with the applications of the entropy or informational principle to the theory of Weber fractions. The two primary premises of the entropy theory of sensory perception were used to obtain the basic equations ( 9 and 12). These equations were further constrained by adopting, as an approximation, Fechner's conjecture of constant subjective magnitude of the jnd.

Neither of the fundamental equations ( 9 and 12) was formulated ad hoc, specifically to describe the Weber fraction; both had been derived previously as part of a general information theoretical approach to the study of perception. The aim of the paper should be seen in this light: It is an attempt to incorporate the Weber fraction into a general theory of psychophysics rather than to account for fine nuances and detail.

The derived form of the jnd function, as given by Equation 21 , with $\Delta \Psi$ taken as constant, is that of a curve that begins high for low values of $\Phi$ and descends to a plateau. The constraint of constant $\Delta \Psi$ was used throughout this paper because it made possible many numerical approximations. However, at least for audition, we know that $\Delta \Psi$ is not constant, but seems to increase with increasing $\Psi$ (Stevens, 1936). It is not known if this change in the subjective magnitude of the jnd is present in other modalities. If the jnd is changing in magnitude, it can be seen from Equation 21 that a true plateau in the Weber fraction cannot exist. Rather, for large $\Phi$, as $\Delta \Psi$ increases, the Weber fraction will bend upward away from the plateau. This phenomenon is often observed, although not obviously with intensities of sound.

It has been demonstrated, within this theory of perception, that information transmitted per stimulus enters explicitly into most equations as a fundamental psychophysical variable. It has been shown theoretically that the exponent of the power law of sensation is related to the logarithm of the stimulus range very nearly as a rectangular hyperbola, as discovered earlier by Teghtsoonian (1971) using data assembled by Poulton (1967). The parameter governing the shape of that hyperbola was derived nearly completely from a priori principles. This hyperbola was shown to be, more exactly, a curve drawn on a three-dimensional surface (Figure 4). Maximum information per stimulus is given as a function of exponent and log stimulus range. Given any two variables we can, in principle (and within the limits of the definition of "range"), predict the third.

An attempt was made to test the accuracy of the various equations derived, using data that were present in the existing literature, but the test was not conclusive. A proper test of these equations can be made only by measuring, in one group of subjects, all the required variables. This provides rather a challenge to experimenters, since they must be proficient in the execution of three different types of experiment: magnitude estimation, difference limens, and categorical judgments. However, the rewards may be ample. The establishment or refinement of these equations would lead us a little closer to a unified theory of psychophysics, a set of laws deduced logically from a single set of premises, governing all or most psychophysical events.

Equation 16, the time-dependent form of the entropy equation, is a step toward such a unified theory. In principle, this equation governs all psychophysical experiments and phenomena that involve steady stimuli of the intensity type and deal with relationships among the variables stimulus magnitude, subjective magnitude, and stimulus dura-

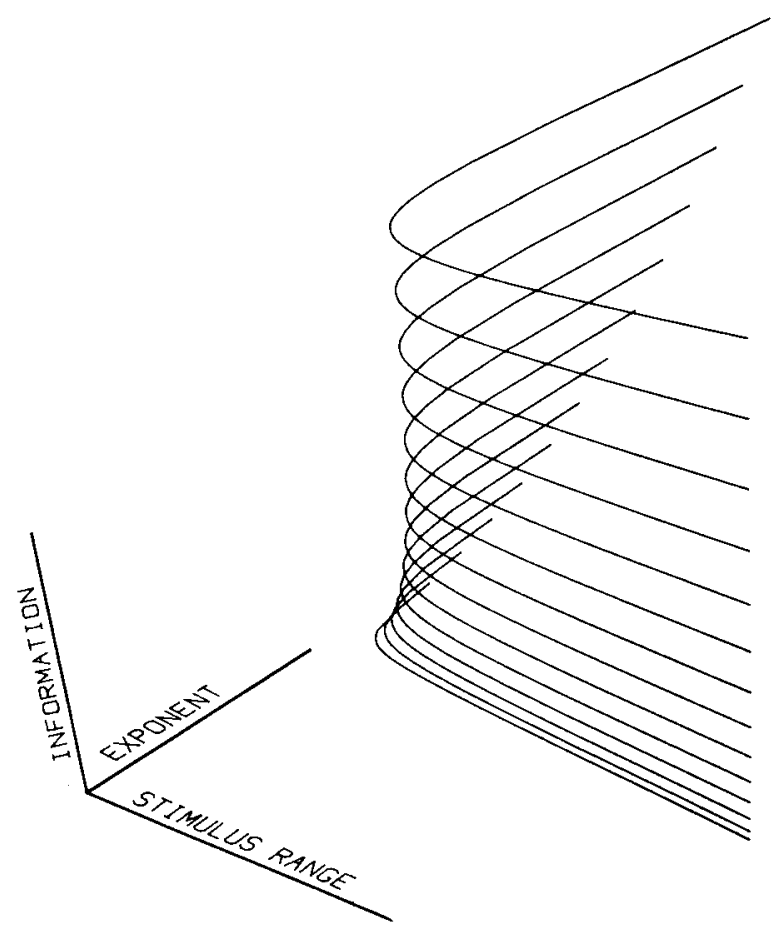

Figure 4. It can be seen from Equation 30 that the product (1.66)(exponent)( $\log _{10}$ stimulus range) is equal, in theory, to the information content of the stimulus. If this quantity of information were exactly the same for all sensory systems, then all measured psychophysical data would lie on one of the rectangular hyperbolas given by (exponent)(log stimulus range) $=$ a constant, depicted in this three-dimensional graph. Because the information transmitted per stimulus varies from system to system, tending to be lower for the chemical senses, the data will lie in a plane that is not quite horizontal in the diagram and that will intersect more than one of the horizontal hyperbolas. 
tion. From this single equation with three parameters, $k$, $n$, and $\beta^{\prime}$, we have derived the laws of Fechner and Stevens, the adaptation function, many relations involving the information transmitted by a stimulus, the mathematical function for Weber's fraction (the "jnd function"), and the stimulus-range equation of Teghtsoonian.

Perhaps of greatest importance, we have accumulated substantial evidence in support of the fundamental premise that perception is the process wherein the (BoltzmannShannon) entropy of a stimulus is reduced.

\section{REFERENCES}

Atkinson, W. H. (1982). A general equation for sensory magnitude. Perception \& Psychophysics, 31, 26-40.

ATTNEAVE, F. (1959). Applications of information theory to psychology. New York: Holt, Rinehart \& Winston.

BarLow, H. W. (1964). The physical limits of visual discrimination. In A. C. Giese (Ed.), Photophysiology (Vol. 2). New York: Academic Press.

Beebe-Center, J. G., Rogers, M. S., \& O'Connell, D. N. (1955). Transmission of information about sucrose and saline solutions through the sense of taste. Joumal of Psychology, 39, 157-160.

Coren, S., Porac, C., \& WARD, L. M. (1984). Sensation and perception (2nd ed.). Orlando: Academic Press.

Ekman, G. (1959). Weber's law and related functions. Joumal of Psychology, 47, 343-352.

Falmagne, J. C. (1974). Foundations of Fechnerian psychophysics. In D. H. Krantz, R. C. Atkinson, R. D. Luce, \& P. Suppes (Eds.), Contemporary developments in mathematical psychology (Vol. 2). San Francisco: W. H. Freeman.

GARNER, W. R. (1953). An informational analysis of absolute judgments of loudness. Journal of Experimental Psychology, 46, 373-380.

GARNER, W. R. (1962). Uncertainty and structure as psychological concepts. New York: Wiley.

GARNER, W. R., \& HAKE, H. W. (1951). The amount of information in absolute judgments. Psychological Review, 58, 445-459.

GrEeN, D. M., \& LUCE, R. D. (1974). Counting and timing mechanisms in auditory discrimination and reaction time. In D. H. Krantz, R. C. Atkinson, R. D. Luce, \& P. Suppes (Eds.), Contemporary developments in mathematical psychology (Vol. 2). San Francisco: W. H. Freeman.

HeChT, S. (1934). Vision: II. The nature of the photoreceptor process. In C. Murchison (Ed.), A handbook of general experimental psychology (pp. 704-828). Worcester, MA: Clark University Press.

Holway, A. H., HuRviCh, L. M. (1937). Differential gustatory sensitivity to salt. American Journal of Psychology, 49, 37-48.

KNUDSEN, V. O. (1923). The sensibility of the ear to small differences of intensity and frequency. Physical Review, Series 2, 21, 84-102.

Luce, R. D., \& Galanter, E. (1963). Discrimination. In R. D. Luce (Ed.), Handbook of mathematical psychology (Vol. 1, pp. 191-243). New York: Wiley.

MANSFIELD, R. J. W. (1973). Brightness function: Effect of area and duration. Joumal of the Optical Society of America, 63, 913-920.

McBride, R. L. (1983a). Category scales of sweetness are consistent with sweetness-matching data. Perception \& Psychophysics, 34, 175-179.

McBride, R. L. (1983b). Psychophysics: Could Fechner's assumption be correct? Australian Journal of Psychology, 35, 85-88.

Meiselman, H. L., Bose, H. E., \& NyKvist, W. F. (1972). Magnitude production and magnitude estimation of taste intensity. Perception \& Psychophysics, 12, 249-252.

Miller, G. A. (1947). Sensitivity to changes in the intensity of white noise and its relation to masking and loudness. Joumal of the Acoustical Society of America, 19, 609-619.

NoRWICH, K. H. (1977). On the information received by sensory receptors. Bulletin of Mathematical Biology, 39, 453-461.

NoRWICH, K. H. (1978). An hypothesis on information, memory and perception. Medical Hypotheses, 4, 156-164.
Norwich, K. H. (1981a). The magical number seven: Making a "bit" of "sense." Perception \& Psychophysics, 29, 409-422.

NoRwich, K. H. (1981b). Uncertainty in physiology and physics. Bulletin of Mathematical Biology, 43, 141-149.

NoRWICH, K. H. (1982). Perception as an active process. Mathematics and Computers in Simulation, 24, 535-539.

NoRwICH, K. H. (1983). To perceive is to doubt: The relativity of perception. Journal of Theoretical Biology, 102, 175-190.

NoRWICH, K. H. (1984). The psychophysics of taste from the entropy of the stimulus. Perception \& Psychophysics, 35, 269-278.

NutTiNG, P. G. (1907). The complete form of Fechner's law. Bulletin of the Bureau of Standards, 3 , No. 1.

Poulton, E. C. (1967). Population norms of top sensory magnitudes and S. S. Stevens' exponents. Perception \& Psychophysics, 2, 312-316.

RHODE, W. S. (1971). Observations of the vibration of the basilar membrane in squirrel monkeys using the Mossbauer technique. Journal of the Acoustical Society of America, 49, 1218-1231.

RIEsz, R. R. (1928). Differential intensity sensitivity of the ear for pure tones. Physical Review, 31, 867-875.

ShANNON, C. E. (1948). A mathematical theory of communication. Bell System Technical Joumal, 27, 623-656.

STEVENS, S. S. (1936). A scale for the measurement of psychological magnitude: Loudness. Psychological Review, 43, 405-416.

STEVENS, S. S. (1970). Neural events and the psychophysical law. Science, 170, 1043-1050.

Teghtsoonian, R. (1971). On the exponents in Stevens' law and the constant in Ekman's law. Psychological Review, 78, 71-80.

\section{NOTES}

1. The jnd, or just noticeable difference, is defined very clearly by Coren, Porac, and Ward (1984). Following their definition, we may say that if a pair of stimuli are separated in physical magnitude by $\Delta \Phi$, the subject will be able to detect the difference between them about half of the time. We shall adhere to this definition except in a single instance, which is discussed in the section on evaluation of the equations.

2. For the three modalities tested in this paper (taste, visual, and auditory intensity), the number of jnds spanned by the plateau of the Weber fraction constitutes about $90 \%$ of the total number of jnds under the total Weber fraction curve, using Equation 24 or Equation 25 . That is, $\Psi / \Delta \Psi$, where $\Psi$ is the subjective magnitude at the extreme right-hand side of the plateau region and $\Delta \Psi$ is the magnitude of the jnd (assumed constant), gives a good approximation of the total number of jnds between threshold and $\Phi_{m}$, the maximum physiological stimulus.

3. Equation $A 4$ can be rearranged algebraically to give

$$
\Psi_{m}=(k / 2)\left[\ln \left(1+\beta \Phi^{n}\right)\right]_{\Phi_{0}}^{\Phi_{m}}
$$

as required by Equation 12. All we have really done is to differentiate and then integrate again. However, Equation A4 is in a form that can be evaluated or tested from measured Weber fractions.

\section{APPENDIX}

An equation relating the information content of a stimulus with the Stevens exponent, utilizing the extremes of the Weber fraction (Equation 32 of the main text):

Since $\Delta \Psi$ is the subjective magnitude of one jnd, and $d \Phi / \Delta \Phi$ is the number of jnds contained within the element $d \Phi$, therefore $(d \Phi / \Delta \Phi) \cdot \Delta \Psi$ equals the subjective magnitude of this number of jnds. Hence, if $\Phi_{0}$ is the threshold intensity and $\Phi_{m}$ is the $\max$ imum intensity,

$$
\Psi_{m}-\Psi_{0}=\int_{\Phi_{0}}^{\Phi_{m}} \frac{d \Phi}{\Delta \Phi} \Delta \Psi=\begin{aligned}
& \text { Difference in subjective magnitude } \\
& \text { between a stimulus of maximum } \\
& \text { intensity, } \Phi_{m}, \text { and one of threshold } \\
& \text { intensity, } \Phi_{0} .
\end{aligned}
$$


From Equation 21,

$$
\frac{\Delta \Phi}{\Phi}=\frac{2 \Delta \Psi}{n \beta k}\left(\beta+\Phi^{-n}\right) .
$$

Rearranging this equation, multiplying through by $d \Phi$, and integrating,

$$
\int_{\Phi_{0}}^{\Phi_{m}} \frac{d \Phi}{\Delta \Phi} \Delta \Psi=\frac{n \beta k}{2} \int_{\Phi_{0}}^{\Phi_{m}} \frac{d \Phi}{\Phi\left(\beta+\Phi^{-n}\right)} .
$$

Taking $\Psi_{0}$ equal to zero (threshold subjective magnitude) and combining Equation A1 with Equation A2,

$$
\Psi_{m}=\frac{n \beta k}{2} \int_{\Phi_{0}}^{\Phi_{m}} \frac{d \Phi}{\Phi\left(\beta+\Phi^{-\pi}\right)} .
$$

Integrating in Equation $\mathbf{A 3}$, we obtain ${ }^{3}$

$$
\Psi_{m}=\frac{n \beta \mathrm{k}}{2}\left[\frac{1}{\beta} \ln \Phi+\frac{1}{\beta n} \ln \left(\beta+\Phi^{-n}\right)\right]_{\Phi_{0}}^{\Phi_{m}} .
$$

From Equation 19,

$$
H_{m}=\Psi_{m} / k,
$$

so that

$$
H_{m}=\frac{n}{2}\left[\ln \Phi+\frac{1}{n} \ln \left(\beta+\Phi^{-n}\right)\right]_{\Phi_{0}}^{\Phi_{m}} .
$$

We now recognize that, using only the first term on the righthand side of Equation A6, we can obtain, as an approximation, Equation 30.

Let us examine the second term in more detail. Returning first to Equation 21,

$$
\text { Weber fraction, } \begin{aligned}
S & =\frac{\Delta \Phi}{\Phi} \\
& =\frac{2 \Delta \Psi}{n k}+\frac{2 \Delta \Psi}{n \beta k} \Phi^{-n} \\
& =\frac{2 \Delta \Psi}{n k}+\frac{2 \Delta \Psi}{n \beta k \Phi_{0}^{n}}\left(\frac{\Phi_{0}}{\Phi}\right)^{n}
\end{aligned}
$$

and, following Riesz (1928), set

$$
S_{\infty}=2 \Delta \Psi / n k
$$

and

$$
S_{0}-S_{\infty}=2 \Delta \Psi / n \beta k \Phi_{0}^{n} .
$$

Then

$$
S=\frac{\Delta \Phi}{\Phi}=S_{\infty}+\left(S_{0}-S_{\infty}\right)\left(\frac{\Phi_{0}}{\Phi}\right)^{n} .
$$

Clearly, $S=S_{\infty}$ for large $\Phi$, and $S=S_{0}$ for $\Phi=\Phi_{0} . S_{\infty}$ is, therefore, the plateau value of the Weber fraction, if it exists, and $S_{0}$ is its near-threshold value. Recall that when $S_{0}$ is large, Equation 21 may not be completely valid, since $\Delta \Phi$ has been represented by $d \Phi$.

The second term on the right-hand side of Equation A6 contains the expression $\ln \left[\left(\beta+\Phi_{m}^{-n}\right) /\left(\beta+\Phi_{o}^{-n}\right)\right]$. If $\Phi_{m}^{-n} \ll \beta$, then

$$
\ln \frac{\beta+\Phi_{m}^{-n}}{\beta+\Phi_{0}^{-\pi}} \cong \ln \frac{\beta}{\beta+\Phi_{0^{-n}}^{n}}=-\ln \left(\dot{1}+\frac{1}{\beta \Phi_{0}^{n}}\right) .
$$

But from Equations $\mathrm{A} 8$ and $\mathrm{A} 9$,

$$
\frac{S_{0}-S_{\infty}}{S_{\infty}}=\frac{1}{\beta \Phi_{0}^{n}},
$$

that is,

$$
1+\frac{1}{\beta \Phi_{0}^{n}}=\frac{S_{0}}{S_{\infty}} .
$$

Hence, in Equation A11,

$$
\ln \frac{\beta+\Phi_{m}^{-n}}{\beta+\Phi_{0}^{-n}} \cong-\ln \left(\frac{S_{0}}{S_{\infty}}\right) .
$$

Combining Equation A6 with Equation A14,

$$
H_{m}=\frac{n}{2} \ln \frac{\Phi_{m}}{\Phi_{0}}-(1 / 2) \ln \left(\frac{S_{0}}{S_{\infty}}\right) .
$$

Converting to bits of information,

$$
H_{m}=\frac{n}{2 \ln 2} \ln \frac{\Phi_{m}}{\Phi_{0}}-\frac{1}{2 \ln 2} \ln \left(\frac{S_{0}}{S_{\infty}}\right),
$$

which is Equation 32 of the main text.

Equation A16, then, provides an expression for the stimulus information which does not require delineation of the plateau region of the graph. In the hypothetical case where the Weber fraction is constant for all $\Phi$, so that $S_{0}=S_{\infty}$, Equation A16 would be identical with Equation 30 .

(Manuscript received March 20, 1986; revision accepted for publication March 18, 1987.) 